\title{
COCONUT online: Collection of Open Natural Products database
}

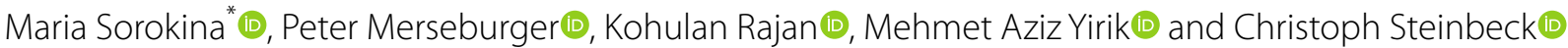

\begin{abstract}
Natural products (NPs) are small molecules produced by living organisms with potential applications in pharmacology and other industries as many of them are bioactive. This potential raised great interest in NP research around the world and in different application fields, therefore, over the years a multiplication of generalistic and thematic NP databases has been observed. However, there is, at this moment, no online resource regrouping all known NPs in just one place, which would greatly simplify NPs research and allow computational screening and other in silico applications. In this manuscript we present the online version of the COlleCtion of Open Natural prodUcTs (COCONUT): an aggregated dataset of elucidated and predicted NPs collected from open sources and a web interface to browse, search and easily and quickly download NPs. COCONUT web is freely available at https://coconut.naturalproducts.net.
\end{abstract}

Keywords: Natural products, Database, NoSQL, MongoDB, Molecular similarity search, Molecular substructure search

\section{Introduction}

Natural products (NPs) have received constant attention from the scientific community due to their relevance in drug discovery, chemical ecology and molecular biology in general. In a recently published review on NPs databases [1] we inventoried over 120 natural products databases that have been published and used in the last 20 years. However, $16 \%$ of these are not available online anymore, $40 \%$ are commercial and their content cannot be easily accessed. The open resources are generally either specialized on a particular type of NPs, either lack annotations. For instance, the catalog of NPs from the ZINC database [2] is composed of over 80,000 entries, some of which can be purchased, but apart from their structure and that they are from natural origin, no additional information is provided. Super Natural II [3] is considered as the largest among all the NP databases, is accessible online in 2020, but it seems not to be maintained anymore and is mainly composed of compounds

*Correspondence: maria.sorokina@uni-jena.de

Institute for Inorganic and Analytical Chemistry, University Friedrich-

Schiller, Lessing Strasse 8, 07743 Jena, Germany that can be purchased. Another recent database, NPAtlas [4], is constantly growing and extremely well annotated, but it is focusing on microbial NPs only. Another major NPs category, plant-produced compounds, also called phytochemicals, is available in several popular and well maintained databases, such as NuBBEDB [5], KnapSack [6], CMAUP [7] and TCM@Taiwan [8]. In addition to these relatively big databases, there is a plethora of smaller, more specialized NPs collections, such as FooDB [9], a user-friendly database hosting a relatively large number of NPs that are found in food. There is, therefore, a need for a generalistic NPs database, that will efficiently aggregate NPs information from various sources, improve its annotation and offer a pleasant user experience. With this ultimate goal in mind, we first assembled the most complete up-to-date COlleCtion of Open Natural ProdUcTs (COCONUT) that we have been continuously curating and annotating. Studies [10,11] showed that fragments from NPs present in COCONUT have high diversity and structural complexity, which makes it, among other possible applications, a suitable source for drug discovery and can be included in drug design pipelines. Our next step was to make this data available to the scientific community as a full-fledged online natural

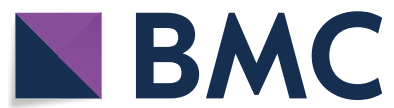

(c) The Author(s) 2021. This article is licensed under a Creative Commons Attribution 4.0 International License, which permits use, sharing, adaptation, distribution and reproduction in any medium or format, as long as you give appropriate credit to the original author(s) and the source, provide a link to the Creative Commons licence, and indicate if changes were made. The images or other third party material in this article are included in the article's Creative Commons licence, unless indicated otherwise in a credit line to the material. If material is not included in the article's Creative Commons licence and your intended use is not permitted by statutory regulation or exceeds the permitted use, you will need to obtain permission directly from the copyright holder. To view a copy of this licence, visit http://creativeco mmons.org/licenses/by/4.0/. The Creative Commons Public Domain Dedication waiver (http://creativecommons.org/publicdomain/ zero/1.0/) applies to the data made available in this article, unless otherwise stated in a credit line to the data. 
products database, maintained at https://coconut.natur alproducts.net.

The COCONUT database is free and open to all users and there is no login required to access it. Its web interface allows diverse simple searches (e.g. by molecule name, InChI, InChI key, SMILES, drawn structure, molecular formula), advanced search by molecular features, together with substructure and similarity searches. Users can also download the whole dataset or search results in different formats. The database can be queried programmatically via a REST API, which facilitates COCONUT integration in workflows. The web interface, the back-end and the database are deployed as Docker containers, making it easily portable for hosting other sets of NPs and to be deployed on local installations.

\section{Construction and content}

COCONUT is assembled from a large number of chemical data sources (Table 1), from which NPs have been thoroughly extracted, curated, processed and annotated. The resulting NPs collection is presented within the fullfledged chemical database developed specially for this purpose (Fig. 1). Data curation and processing steps, together with the construction of the web interface and the description of available features are described below.

\section{Data provenance, model and content}

COCONUT data has been extracted from 53 various data sources and several manually collected from literature sets, as shown in Table 1. In the current COCONUT release (October 2020), there are 406,076 unique "flat" (with no stereochemistry) NPs, and a total of 730,441
NPs where stereochemistry has been preserved when available.

Every molecule collected from external sources passed a quality control and a registration procedure, where its structure is checked for size (between 5 and 210 heavy atoms), connectivity (only the biggest connected structure is kept), presence of pseudo-atoms, if implicit and explicit hydrogens are correct, and if the bonds are correct and the valences are conserved. The Kekule representation is also assigned to the aromatic systems of each compound. At this step, tautomers and ionisation states are standardized following the ChEMBL chemical structure curation pipeline [12].

Then, NPs from different provenance are unified based on the identity of their InChI keys without stereochemistry. This unification step is performed without stereochemistry, as in different data sources stereochemistry is not systematically present and can also be represented differently. When available, the original molecular structure with stereochemistry is preserved and can be accessed for each NP entry.

The authors are well aware that different stereoisomers of a compound can have very different biological activity. The procedure described above was a necessary step to create a unified resource out of distributed databases of varying quality. Further curation will gradually improve stereochemical assignments and linkage to original source articles.

Each unique NP is then assigned a unique identifier, composed of the "CNP" prefix and 7 digits. An automatic curation for NP metadata is performed, comprising the retrieval of its official name, synonyms, cross-references

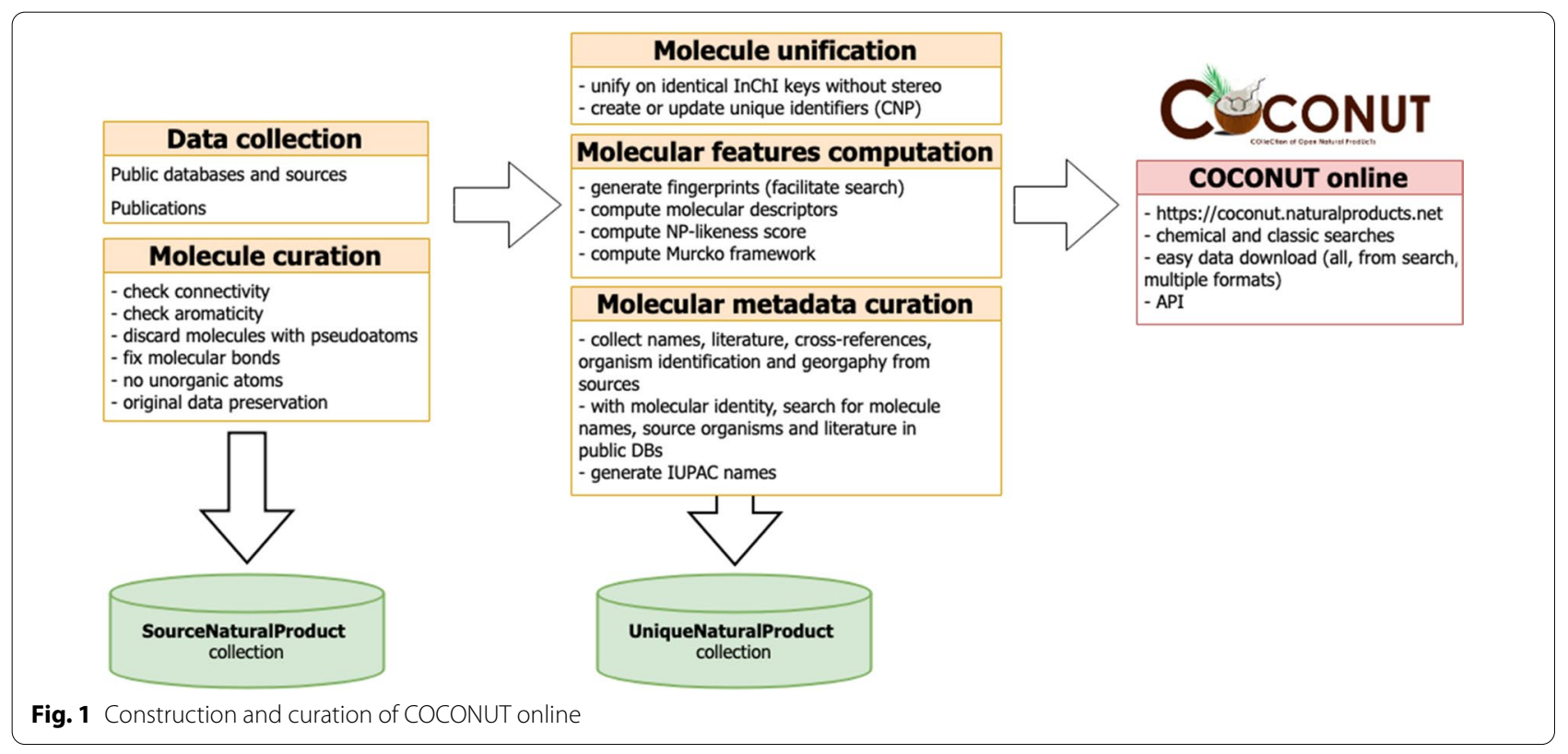


Table 1 Public databases and datasets from which COCONUT was assembled

\begin{tabular}{|c|c|c|}
\hline $\begin{array}{l}\text { Database name ("NP" suffix is added to database } \\
\text { name when only a subset of it contains natural } \\
\text { products) }\end{array}$ & $\begin{array}{l}\text { Number of entries integrated } \\
\text { in COCONUT from the source }\end{array}$ & Most resent publication or resource URL \\
\hline AfroCancer & 365 & [33] \\
\hline AfroDB & 874 & [34] \\
\hline AfroMalariaDB & 252 & [35] \\
\hline AnalytiCon Discovery NPs & 4908 & {$[36]$} \\
\hline BIOFACQUIM & 400 & [37] \\
\hline BitterDB & 625 & [38] \\
\hline Carotenoids Database & 986 & [39] \\
\hline ChEBI NPS & 14603 & [20] \\
\hline ChEMBL NPS & 1585 & [21] \\
\hline ChemSpider NPs & 9027 & {$[40]$} \\
\hline CMAUP (cCollective molecular activities of useful plants) & 20868 & [7] \\
\hline ConMedNP & 2504 & [41] \\
\hline ETM (Ethiopian Traditional Medicine) DB & 1633 & [42] \\
\hline Exposome-explorer & 478 & [43] \\
\hline FooDB & 22123 & [9] \\
\hline $\begin{array}{l}\text { GNPS (Global Natural Products Social Molecular Network- } \\
\text { ing) }\end{array}$ & 6740 & [44] \\
\hline HIM (Herbal Ingredients in-vivo Metabolism database) & 962 & [45] \\
\hline HIT (Herbal Ingredients Targets) & 470 & [46] \\
\hline Indofine Chemical Company & 46 & [47] \\
\hline InflamNat & 536 & [48] \\
\hline InPACdb & 122 & [49] \\
\hline InterBioScreen Ltd & 67291 & {$[50]$} \\
\hline KNApSaCK & 44422 & [6] \\
\hline Lichen Database & 1453 & [51] \\
\hline Marine Natural Products & 11880 & {$[52]$} \\
\hline Mitishamba database & 1010 & [53] \\
\hline NANPDB (Natural Products from Northern African Sources) & 3914 & [54] \\
\hline NCI DTP data & 404 & {$[55]$} \\
\hline NPACT & 1453 & {$[56]$} \\
\hline NPASS & 27424 & {$[57]$} \\
\hline NPAtlas & 23914 & [4] \\
\hline NPCARE & 1362 & [58] \\
\hline NPEdia & 16166 & [59] \\
\hline NUBBEDB & 2022 & [5] \\
\hline p-ANAPL & 467 & {$[60]$} \\
\hline Phenol-explorer & 681 & [61] \\
\hline PubChem NPs & 2828 & [27] \\
\hline ReSpect & 699 & [62] \\
\hline SANCDB & 592 & [63] \\
\hline Seaweed Metabolite Database (SWMD) & 348 & [64] \\
\hline Specs Natural Products & 745 & [65] \\
\hline Spektraris NMR & 242 & [66] \\
\hline StreptomeDB & 6058 & [67] \\
\hline Super Natural II & 214420 & [3] \\
\hline TCMDB@Taiwan (Traditional Chinese Medicine database) & 50862 & [8] \\
\hline TCMID (Traditional Chinese Medicine Integrated Database) & 10552 & [68] \\
\hline TIPdb (database of Taiwan indigenous plants) & 7742 & [69] \\
\hline
\end{tabular}


Table 1 (continued)

\begin{tabular}{lcc}
\hline $\begin{array}{l}\text { Database name ("NP" suffix is added to database } \\
\text { name when only a subset of it contains natural } \\
\text { products) }\end{array}$ & $\begin{array}{l}\text { Number of entries integrated } \\
\text { in COCONUT from the source }\end{array}$ & Most resent publication or resource URL \\
\hline TPPT (Toxic Plants-PhytoToxins) & 1483 & {$[70]$} \\
UEFS (Natural Products Databse of the UEFS) & 481 & {$[71]$} \\
UNPD (Universal Natural Products Database) & 156865 & {$[72]$} \\
VietHerb & 4759 & {$[73]$} \\
ZINC NP & 67327 & {$[74]$} \\
Manually selected molecules & 61 & $\times$ \\
\hline
\end{tabular}

to other major chemical databases. Then, a range of molecular properties, descriptors and fingerprints (full list in Table 2) are computed using the in-build CDK [13] libraries. As the number of the computed properties is quite big (73 fields in each document corresponding to one unique NP), only a selected fraction of them is displayed on the COCONUT web interface. Finally, the first round of automatic curation of NP metadata, in particular the molecular name synonyms, cross-references with other major chemical databases, correction of the literature references (PubMed identifiers and DOIs) and taxonomy is performed. All original data, unified NPs and the derived and calculated information are stored in MongoDB. The chemical classification of all NPs in COCONUT is performed with ClassyFire [14], and, when available, is displayed in the corresponding section of the compound page. ClassyFire provides a hierarchical chemical classification of chemical compounds and enables grouping NPs by their chemical class. Additionally, frameworks facilitating NPs analyses for their chemical and therapeutic properties are computed for NPs, such as Murcko frameworks [15], Ertl Functional Groups [16] and deepSMILES [17]. DeepSMILES is an adaptation of SMILES for use in deep machine learning of chemical structures. Due to the increased usage of deep learning in chemistry, it is indeed interesting to provide this new chemical representation type pre-computed for NPs.

Last, the annotation level of each NP in COCONUT is computed. It is a 5-star-based system, where 1 star is the lowest annotation quality (no verified common name, no taxonomic provenance annotation, no literature reference and no trusted data source) and 5 stars is the highest quality, with all the intermediate annotation qualities reflected by 2, 3 and 4 stars. Only ChEBI [18], KNApSAcK [6], ChEMBL [19], CMAUP [7], NPAtlas [4] and, of course, the manually picked data are considered as trusted data provenances. For example, caryolivine (CNP0235854) has a 5-stars annotation because it has a verified common name, is known to be produced by Caryomene olivascens, a plant, is associated to a scientific publication and is present in KNApSAcK. The COCONUT NP CNP0330764 has no verified common name, only a computed IUPAC one, but is present in CMAUP and is known to be produced by a range of plants, therefore it's annotation level is 3. The annotation level is represented with stars on the NP page.

\section{Natural product naming}

NPs common names in COCONUT have been retrieved, when available, from their databases of origin. The remaining NPs have been searched by InChI in major chemical databases (PubChem, ChEMBL and ChEBI) and common names and synonyms were retrieved when occurences the compound were found. Additionally to this, IUPAC names were computed for all COCONUT NPs using ChemAxon's MolCovert [20], to add nomenclature homogeneity to the dataset. Furthemore, the IUPAC names are used as the main NP name when no official chemical name has been found nor in the original sources, nor by searching big chemical databases. Therefore, all NPs in COCONUT have at least one molecular name.

\section{Computed molecular features}

Figure 2 demonstrates the distributions and relationships of a small selection of computed molecular features within COCONUT. Sugar moieties occur frequently in NPs and have an important impact on their bioactivities and physicochemical properties. However, they are often redundant and therefore obstruct the study of the aglycon. For this reason, NPs in COCONUT have been analysed for sugar moieties presence, and a deglycosylated structure representation was made available in the database. Sugar moieties manipulations were performed using the Sugar Removal Utility [21]. To track their influence on other features, their absence and presence are colour-mapped (no sugar moiety in the molecular structure in blue, and the presence of at least one sugar moiety in orange). The wide molecular weight range is typical for NPs; it is, however, interesting to notice its 
Table 2 Molecular features present in COCONUT and in their disponibility in the web interface

\begin{tabular}{|c|c|c|}
\hline Natural product feature & $\begin{array}{l}\text { Field name in MongoDB- } \\
\text { uniqueNaturalProduct collection }\end{array}$ & $\begin{array}{l}\text { Displayed } \\
\text { on the website }\end{array}$ \\
\hline COCONUT identifier & coconut_id & $x$ \\
\hline List of SMILES with stereochemistry and their provenance & absolute_smiles & $x$ \\
\hline AlogP (Ghose-Crippen LogKow) & alogp & $x$ \\
\hline AlogP2 & alogp2 & $x$ \\
\hline AMR_-molar refractivity & amralogp & \\
\hline Annotation level of the NP (from 1 to 5) & annotationLevel & $x$ \\
\hline BCUT decriptor (Eigenvalue based) & bcutDescriptor & \\
\hline Bond number in the NP & bond_count & $x$ \\
\hline BPol descriptor & bpol & $x$ \\
\hline CAS number & cas & $x$ \\
\hline List of literature DOls mentioning the NP & citationDOI & $x$ \\
\hline Boolean_-if the molecule contains linear sugars & contains_linear_sugars & \\
\hline Boolean - if the molecule contains circular sugars & contains_ring_sugars & \\
\hline Boolean-if the molecule contains sugar moieties & contains_sugar & \\
\hline deepSMILES & deep_smiles & $x$ \\
\hline Eccentric Connectivity Index Descriptor & eccentricConnectivityIndexDescriptor & $x$ \\
\hline List of tl Functional Groups & ertlFuntionalFragments & \\
\hline List of Ertl Functional Groups in pseudo SMILES & ert|FunctionalFragmentsPseudoSmiles & \\
\hline FMF descriptor & fmfDescriptor & $x$ \\
\hline List of data sources containing the NP & found_in_databases & \\
\hline Fragment complexity descriptor & fragmentComplexityDescriptor & $x$ \\
\hline List of circular fragments (molecular signatures) of the deglycosylated NP & fragments & \\
\hline List of circular fragments (molecular signatures) of the whole NP & fragmentsWithSugar & \\
\hline Fractional CSP3 Descriptor (non-flatness of a molecule) & fsp3 & $x$ \\
\hline List of continents and regions where the organism producing the NP is found & geoLocation & \\
\hline Gravitational index descriptor (heavy atoms only) & gravitationallndexHeavyAtoms & \\
\hline Hydrogen bond acceptor count & hBondAcceptorCount & \\
\hline Hydrogen bond donor count & hBondDonorCount & \\
\hline Number of heavy atoms in the NP & heavy_atom_number & $x$ \\
\hline Hybridization Ratio Descriptor (fraction of sp3 carbons to sp2 carbons) & hybridizationRatioDescriptor & \\
\hline InChl (without stereochemistry) & inchi & $x$ \\
\hline InChl key & inchikey & $x$ \\
\hline IUPAC name & iupac_name & $x$ \\
\hline First kappa shape index & kappaShapelndex1 & \\
\hline Second kappa shape index & kappaShapelndex2 & \\
\hline Third kappa shape index & kappaShapelndex3 & \\
\hline Number of failures in the Lipinski rule of 5 & lipinskiRuleOf5Failures & $x$ \\
\hline LogP descriptor (Mannhold version) & manholdlogp & \\
\hline Maximal number of rings in the NP & max_number_of_rings & $x$ \\
\hline Minimal number of rings in the NP & min_number_of_rings & $x$ \\
\hline Molecular formula & molecular_formula & $x$ \\
\hline Molecular weight & molecular_weight & $x$ \\
\hline Murcko Framework & murcko_framework & $x$ \\
\hline Official name (when available) & name & $x$ \\
\hline NP-likeness score & npl_score & $x$ \\
\hline NP-likeness score computed on the glycosylated molecule & npl_sugar_score & \\
\hline Total number of carbons & number_of_carbons & $x$ \\
\hline Total number of nitrogens & number_of_nitrogens & \\
\hline Total number of oxygens & number_of_oxygens & \\
\hline
\end{tabular}


Table 2 (continued)

\begin{tabular}{|c|c|c|}
\hline Natural product feature & $\begin{array}{l}\text { Field name in MongoDB- } \\
\text { uniqueNaturalProduct collection }\end{array}$ & $\begin{array}{l}\text { Displayed } \\
\text { on the website }\end{array}$ \\
\hline Number of sporo atoms & numberSpiroAtoms & \\
\hline Petitjean Number Descriptor & petitjeanNumber & $x$ \\
\hline Petitjean geometrical shape index & petitjeanShapeGeom & \\
\hline Petitjean geometrical shape index & petitjeanShapeTopo & \\
\hline PubChem fingerprint in MongoDB BinData format & pubchemBits & \\
\hline PubChem fingerprint as list of booleans & pubchemFingerprint & \\
\hline SMILES with all hydrogen explicit & smiles & $x$ \\
\hline Number of heavy atoms of the deglycosylated moiety & sugar_free_heavy_atom_number & \\
\hline SILES of the deglycosylated moiety & sugar_free_smiles & \\
\hline Total atom number of the deglycosylated moiety & sugar_free_total_atom_number & \\
\hline List of synonym names of the NP & synonyms & $x$ \\
\hline List of NCBI taxonomy identifiers of organisms producing the NP & taxid & \\
\hline List of organisms producing the NP in text form & textTaxa & \\
\hline Topological polar surface area descriptor & topoPSA & \\
\hline Total atom count in the NP (incuding hydrogens) & total_atom_number & \\
\hline Fractional polar surface area descriptor & tpsaEfficiency & \\
\hline Unique SMILES (CDK) & unique_smiles & $x$ \\
\hline Volume descriptor & vabcDescriptor & \\
\hline Vertex adjacency information & vertexAdjMagnitude & \\
\hline Wiener Path Number & wienerPathNumber & $x$ \\
\hline Wiener Polarity Number & wienerPolarityNumber & \\
\hline XLogP descriptor & $x \log p$ & $x$ \\
\hline Cross-references to toher chemical ressources & xrefs & $x$ \\
\hline Zagreb Index & zagreblndex & $x$ \\
\hline Chemical superclass of the NP computed with ClassyFire & chemicalSuperClass & $x$ \\
\hline Chemical class of the NP computed with ClassyFire & chemicalClass & $x$ \\
\hline Chemical subclass of the NP computed with ClassyFire & chemicalSubClass & $x$ \\
\hline Direct parent in the chemical ontology of the NP computed withClassyFire & directParentClassification & $x$ \\
\hline
\end{tabular}

correlation with the number of oxygen atoms in the molecule, regardless of the presence and absence of sugar. Another interesting correlation to be noted is between the molecular weight and the nitrogen atom number in sugar-free molecules. The NP-likeness score [22], trained on high-quality NPs dataset and computed with NaPLeS [23], which was trained on high-quality NPs dataset, has a typical distribution for an NPs set, where most molecules have a positive score.

At this point, an additional NPs curation step has been performed, due to the possible inconsistency in genuine NPs of one of the used sources, SuperNatural II. NPs that are not occuring in other datasets used to assemble COCONUT, but only in SuperNatural II, have been thoroughly tested. To be kept in COCONUT and be considered as a genuine or predicted NP, such a molecule has to have a strictly positive NP-likeness score, be classified as a NP by NPclassifier [24], a deep neural network-based structural classification tool specialised in NPs or have a sugar moiety in its structure. The 24,880 molecules from SuperNatural II that didn't pass this additional quality control have been removed from COCONUT, until further proof of their natural provenance.

Counting rings in a molecule can become a complex task, as the outer perimeter of two fused rings can be counted as one big ring. With more condensed rings, the number of fused ring perimeters (aka as the set of all rings) can grow steeply. In Fig. 2, only the minimal ring count (the minimal cycle base) is represented.

\section{Natural product annotation}

The particularity of NPs, opposed to synthetic molecules, which constitute the biggest part of chemical databases, resides in their production by living organisms. Therefore, in addition to their structure and computable structural properties, NPs need to be annotated with at least one literature reference, mentioning where, when and from which organism the NP was isolated. As 


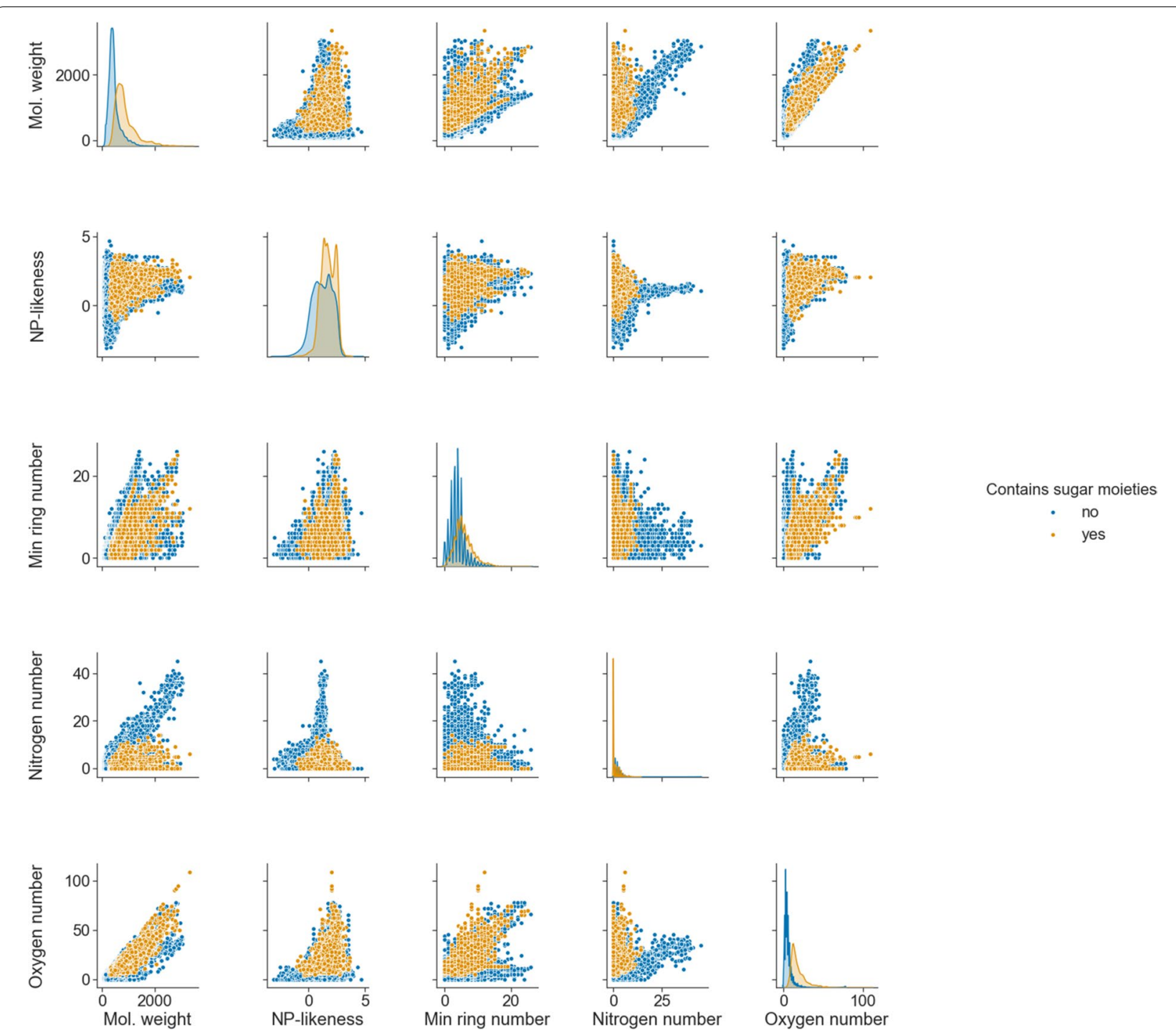

Fig. 2 Pair plot of a selection of five of the molecular features available in COCONUT. Colour mapping corresponds to the presence (yellow) and absence (blue) of glycosidic moieties in the molecular structures of the NPs in COCONUT

a direct consequence, an NP entry should be associated with at least one organism, preferentially with an NCBI taxonomy identifier and the geographic location where the organism is naturally occuring or has been collected. Regrettably, this metadata is often omitted in public databases from which COCONUT was assembled. Therefore, only $31.5 \%(134,379)$ of NPs in COCONUT are annotated with at least one organism taxa, for $15.4 \%(66,068)$ of NPs the geographic location (on the continent level) of the organism occurence or collection is known and only $16.6 \%(70,730)$ of NPs have at least one literature reference. These numbers combine both the retrieval of the original NP annotations from their sources and our efforts to retrieve more extensive information from major trusted chemical databases, PubChem [25], ChEMBL [19], ChEBI [18], CMAUP [7] and KnapSacK [6]. Despite our efforts, most of the links between the original publication of the structure elucidation of an NP and its reference, source organism and its geographical location are still missing. A possible solution to fill these gaps is manual curation, but the amount of data in COCONUT is redhibitory for even considering this approach. Another solution is to use unsupervised machine learning for optical recognition approaches, to parse modern peer-reviewed literature and books to re-establish links between NPs structures and their provenance.

We analysed the taxonomic classification of known NPs producers together with overlaps in NPs production 
between superkingdom for the $31 \%$ of the NPs in COCONUT for which the provenance organism is known (Fig. 3). Here are distinguished five taxonomic categories: plants, bacteria, fungi, animals and marine. The last one is not a proper monoclade classification, but rather reflects a group of organisms that are found only in marine and oceanic environments, and therefore can overlap in terms of its species and NP content with other categories, which are more stringent taxonomically. A large part (65\%) of these annotated NPs are produced only by plants, and only very few $(0.5 \%)$ are from animal origin. Main overlaps in terms of NP production between the taxonomic kingdoms are between plants and marine organisms (which is unsurprising, as there can be real plants among the marine entities) and surprisingly between plants and fungi. The other overlaps between taxonomic kingdoms are not as significant. It needs to be pointed out here that multicellular organisms, such as plants, animals and some of the fungi are most of the time in symbiosis with microorganisms, in particular bacteria. Therefore, NPs isolated from such a multicellular organism can be synthesized and secreted by their symbionts or microbiomes, and therefore mistakenly assigned to an incorrect organism.

The geographic location of the collection or the natural presence of the NP-producing organism is a piece of information that is even more difficult to obtain. Nowadays, a range of organisms, and in particular plants, can be found in different parts of the planet due to globalisation and their success in human consumption (e.g. garlic, tomatoes, curcuma or ginger). It is, therefore, difficult, if not impossible, to determine their original provenance. Also, the geographical information is often omitted in literature and most NPs databases. When available, the geographical provenance is stored in the MongoDB dump of COCONUT, but not displayed on the website.

For NPs where geographical information is available, it appears that most of them are produced by

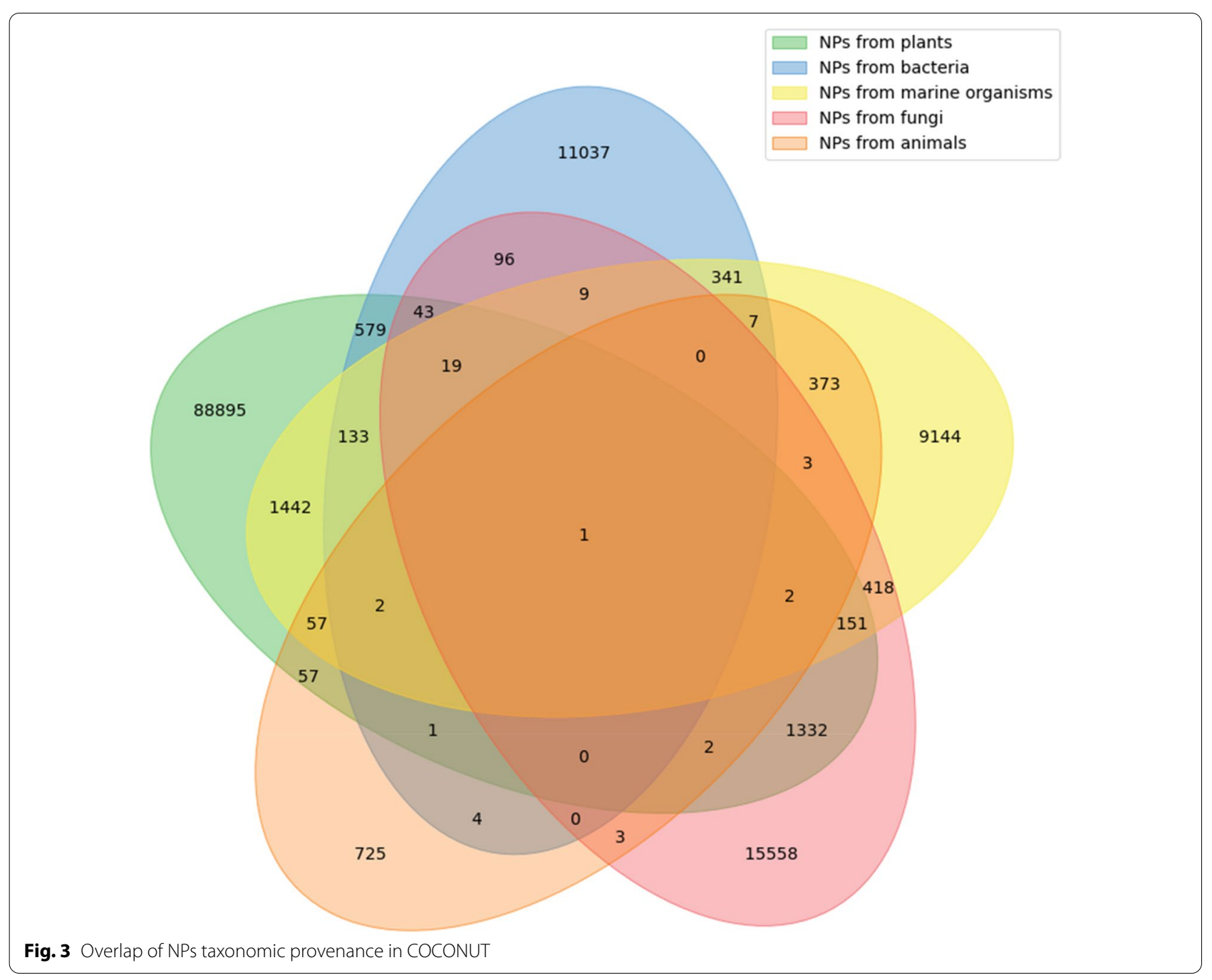


organisms that have been isolated in Asia (Fig. 4). This bias is introduced by the intensive study by scientists of the traditional Chinese and Indian medicines and by the big efforts in isolation and elucidation of NPs from medicinal plants. NPs from the African continent are also well represented in COCONUT (Fig. 4), mainly due to the scientific interest in African traditional medicines and African biodiversity. There is, for now, no data from the biodiversity of the Australian continent, and only very little data for NPs isolated from endemic European organisms. NPs from the Americas are mainly extracted and solved while Brazilian and Mexican biodiversity exploration. Only a few NPs are present in more than one continent, mainly in Asia and Africa, and the overlap values are biased by the very different NPs set sizes between the different continents.

\section{Web interface and technical specificities}

All COCONUT data is stored with MongoDB, a crossplatform document-oriented NoSQL database program.
The smallest unit in MongoDB is a document, composed of key and value pairs that are similar to JSON objects. Documents of the same nature are organized in collections, which are the equivalent of the SQL-based databases tables. MongoDB is particularly adapted to big and complex data, supports multiple indexing, including text indexing allowing enhanced text search in text-indexed fields and contains a wide range of in-build search and analysis functions.

Two major collections are present in the COCONUT database: SourceNaturalProduct, which contains the original NPs data collected from the open sources, and UniqueNaturalProduct, the unified and curated collection of NPs. The full version of COCONUT with all the calculated features can be accessed as a MongoDB dump in the Downloads section of the website. Requests for displaying additional crucial features in the web interface and making them searchable through the advanced search interface are welcome via the COCONUT GitHub tracker (see below).

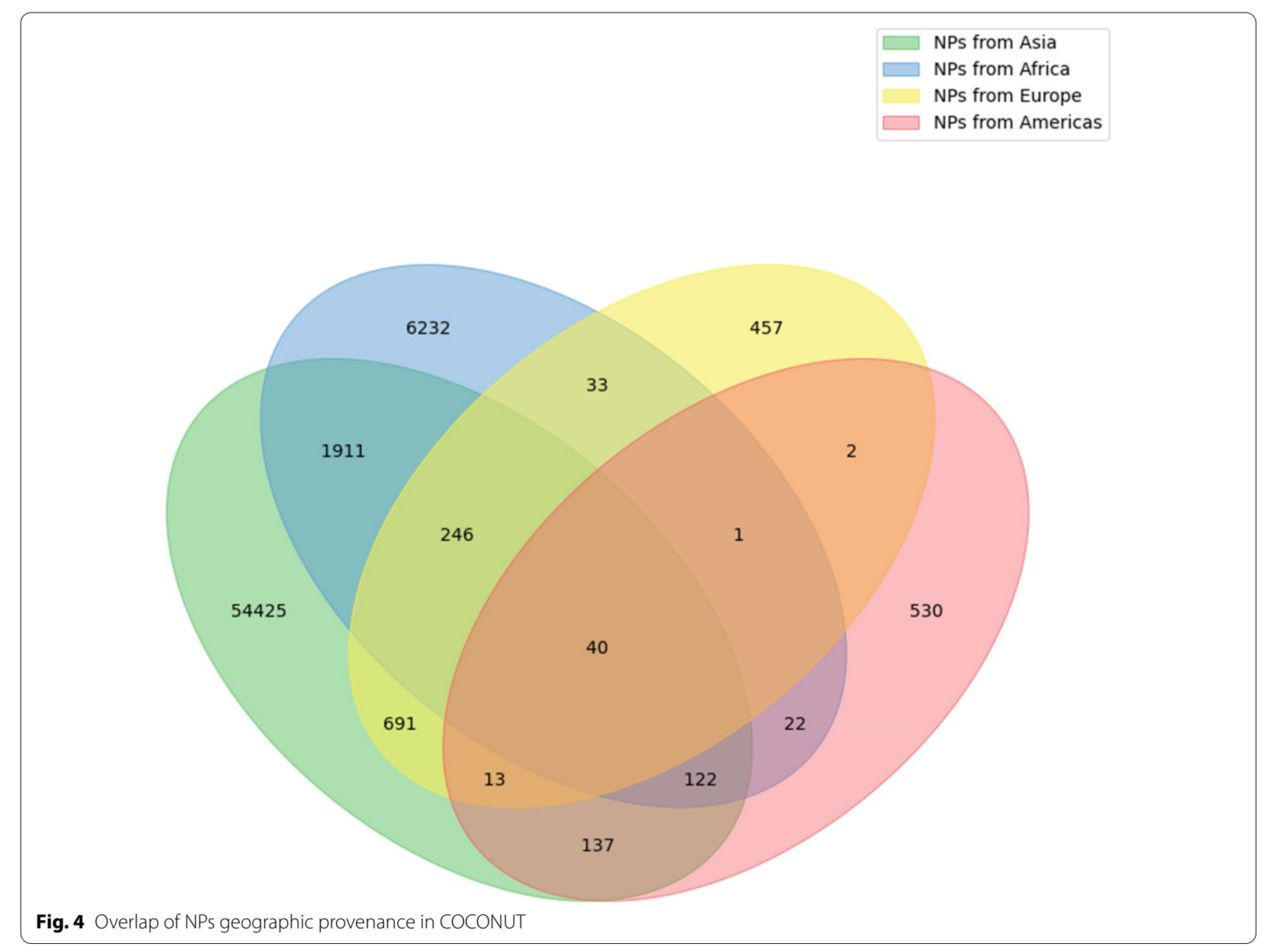


The COCONUT online front-end is developed entirely with React.js [26], a JavaScript library to build responsive and efficient user interfaces. The OpenChemLib library [27] is used to handle the chemical editor for the search functions. The COCONUT back-end, allowing to process the front-end requests and to communicate with the database is written in Kotlin and Java 11 using the Spring framework. The CDK [13] library is used to process chemical information and formats.

COCONUT web interface, back-end and database are entirely Dockerised, allowing a quick and easy deployment on local servers and cloud. All the code, for both front-end and back-end, is available on GitHub (https:// github.com/mSorok/NaturalProductsOnline).

\section{Searching the database}

COCONUT online has been developed to be a fullfledged chemical database and in particular to fit the NPs structural and annotational particularities, with all the subsequent functions. At the moment, the chemical search is uncommon with MongoDB, therefore several approaches have been implemented to run molecular substructure and similarity searches.

\section{Simple search}

The so-called "simple" search can be performed using the header search bar. The users can enter there molecule names (e.g. "curcumin"), SMILES, InChI, InChi key, COCONUT ids and molecular formulas. Name search uses native MongoDB text indexing, allowing fuzzy, flexible search in the "name" and "synonyms" fields. The input string type is first identified using regular expressions, then the DB is queried against the appropriate fields, and the result, when exists, is returned to the front-end.

\section{Substructure search implementation}

Searching for an exact substructure in a MongoDB database of molecules appears to be surprisingly easy. Each molecule in the database needs to have their fingerprints of choice (in COCONUT are used the PubChem fingerprints) to be precomputed and stored as a list of bytes (BinData type in MongoDB). The query molecule (substructure) then needs to have its fingerprint to be also computed and to be matched against the database using the \$allBitsSet function [28]. This native to MongoDB function allows to select documents in a collection where a BinData field has all the query bits set to "on" (but can have bits set to "on" that are not present in the query). To confirm the substructure match, the user can select between the default Ullmann [29], the Vento-Foggia [30] and the depth-first (DF) [31] pattern matching approaches, all performed using the CDK in-build algorithms. These three pattern matching techniques tend to, generally, return very similar results, the difference between them lying rather in their approach to matching substructures, therefore the usage of the default, Ullmann, method is to be privileged by users unfamiliar with the intricacies of such matching.

\section{Similarity search implementation}

Similarity search with MongoDB was implemented following the excellent ChEBML blog post on LSH-based similarity search in MongoDB [32] and adapted to Java, Kotlin and Spring data. In this approach, the MongoDB aggregation framework is used to perform inverted indexing search against PubChem fingerprints stored in a separate table and referencing COCONUT identifiers that contain the molecular features encoded by each bit.

\section{Advanced search}

The advanced search supports searching for NPs in COCONUT according to a range of parameters, such as molecular formula, molecular descriptor values, number of rings, type of sugar moieties present in them, etc.

\section{Querying COCONUT through the API}

A REST API has been developed for COCONUT online in order to permit programmatic querying and facilitate its integration in workflows. It relies on Kotlin API functionalities and it's usage, together with some examples, is described in detail in the documentation section of the website (https://coconut.naturalproducts.net/docum entation).

\section{Documentation}

Complete documentation describing COCONUT, its data and functionalities are available at the documentation section of the website https://coconut.naturalpro ducts.net/documentation.

\section{Utility and discussion}

The online COCONUT database is an open tool for researchers in the natural products community. COCONUT is the biggest collection of NPs in 2020 and the data it contains already benefits researchers in NPs with various aims, such as biodiversity research and drug discovery. The web interface allows querying and parsing the data collection in various, chemically relevant ways with adequate performance. It is also the first big chemical database using MongoDB as a storage management system.

A wide range of molecular descriptors are pre-computed and literature, producer taxonomy and their geography are as much annotated as currently possible without extensive manual curation. The web database can be searched in multiple ways, by molecular structure, by 
compound name and by molecular features, making this repository a complete chemical database. The user interface is modern and easy to use. Besides, the whole content of COCONUT is available for download in multiple formats.

In the close future, COCONUT will support user registration to enable user-driven NPs curation and submission and will undergo a better data annotation, in particular regarding the organisms that are producing the NPs, their geography and the corresponding literature, using deep learning approaches.

\section{Feedback}

Bugs, annotation issues and requests of new COCONUT entries or re-annotation of existing ones can be reported at the project issues tracker (https://github.com/mSorok/ NaturalProductsOnline/issues). Suggestions for new features are also welcome.

\section{Availability}

All COCONUT data, code to process raw NPs data, data quality control and annotation, and the code for the font- and the back-end of the COCONUT online website are freely available without any restriction. The latest COCONUT data, as MongoDB full dump can be downloaded at https://coconut.naturalproducts.net/downl oad. Code for data assembly, processing and quality control process codes is available on GitHub at https://githu b.com $/ \mathrm{mSorok} / \mathrm{COCONUT}$. The code for the front-end and back-end is also available on GitHub at https://githu b.com/mSorok/NaturalProductsOnline.

\section{Conclusions}

COCONUT is the largest open collection of elucidated and predicted NPs at this time. It has a great potential of being of particular importance for the NPs research community as it gathers most of open NPs knowledge in one single place, and makes it easily accessible and queryable.

The final aim of COCONUT is to provide to the scientific community NPs structures and their provenance, i.e.. organisms that synthesize them and geographic location of the latter. However, a lot of data curation, in particular using new generation deep learning-based methods of extracting information from publications and books, together with website functionalities developments are still need to be done for COCONUT, but the database as it is now is already an important tool to facilitate NPs and medicinal chemistry research.

\section{Abbreviations}

NPs: Natural products; COCONUT: COlleCtion of Open Natural ProdUcTs; CDK: Chemistry Development Kit; IUPAC: International Union of Pure and Applied Chemistry; API: Application Programming Interface; REST: REpresentational State Transfer; DOI: Digital Object Identifier; JSON: JavaScript Object Notation; (No)SQL: (non-/not only) Structured Query Language.

\section{Acknowledgements}

Not applicable.

\section{Authors' contributions}

MS coordinated the study, participated in the implementation of the frontend and the backend, collected; processed and curated the COCONUT data and wrote the manuscript. PM participated in the implementation of the front-end and the back-end of COCONUT online. KR and MAY helped with COCONUT data curation. KR designed the COCONUT logo. CS designed and supervised the study. All authors read and approved the final manuscript.

\section{Funding}

Open Access funding enabled and organized by Projekt DEAL. This work was supported by the German Research Foundation within the framework CRC1127 ChemBioSys.

\section{Availability of data and materials}

the source code of the web interface and the back-end is available on GitHub at https://github.com/mSorok/NaturalProductsOnline. The data was curated and processed using the COCONUT code suite available on GitHub at https:// github.com/mSorok/COCONUT. All COCONUT data can be accessed on the website at https://coconut.naturalproducts.net/ and downloaded entirely or partially in several formats (MongoDB dump, SDF and SMI (SMILES)).

\section{Competing interests}

The authors declare no competing interests.

Received: 9 September 2020 Accepted: 23 November 2020

Published online: 10 January 2021

\section{References}

1. Sorokina M, Steinbeck C (2020) Review on natural products databases: where to find data in 2020. J Cheminform 12:20. https://doi.org/10.1186/ s13321-020-00424-9 [cito:citesForInformation]

2. ZINC natural products subset. https://zinc15.docking.org/substances/ subsets/natural-products/. Accessed 19 Nov 2020 [cito:usesDataFrom]

3. Banerjee P, Erehman J, Gohlke B-O, Wilhelm T, Preissner R, Dunkel M (2015) Super Natural II- a database of natural products. Nucleic Acids Res 43:D935-D939. https://doi.org/10.1093/nar/gku886 [cito:usesDataFrom]

4. van Santen JA, Jacob G, Singh AL, Aniebok V, Balunas MJ, Bunsko D et al (2019) The natural products atlas: an open access knowledge base for microbial natural products discovery. ACS Cent Sci 5:1824-1833. https:// doi.org/10.1021/acscentsci.9b00806 [cito:usesDataFrom]

5. Pilon AC, Valli M, Dametto AC, Pinto MEF, Freire RT, Castro-Gamboa I (2017) NuBBEDB: an updated database to uncover chemical and biological information from Brazilian biodiversity. Sci Rep 7:7215. https://doi. org/10.1038/s41598-017-07451-x [cito:usesDataFrom]

6. Nakamura K, Shimura N, Otabe Y, Hirai-Morita A, Nakamura Y, Ono N (2013) KNApSAcK-3D: a three-dimensional structure database of plant metabolites. Plant Cell Physiol 54:e4-e4. https://doi.org/10.1093/pcp/ pcs186 [cito:usesDataFrom]

7. Zeng X, Zhang P, Wang Y, Qin C, Chen S, He W (2019) CMAUP: a database of collective molecular activities of useful plants. Nucleic Acids Res 47:D1118-27 [cito:usesDataFrom]

8. Chen CY-C (2011) TCM Database: the World's Largest Traditional Chinese Medicine Database for Drug Screening in silico. PLOS ONE 6:e15939. https://doi.org/10.1371/journal.pone.0015939 [cito:usesDataFrom]

9. FooDB. http://foodb.ca/. Accessed 3 Oct 2019 [cito:usesDataFrom]

10. Chávez-Hernández AL, Sánchez-Cruz N, Medina-Franco JL (2020) A fragment library of natural products and its comparative chemoinformatic 
characterization. Mol Inform 39:2000050. https://doi.org/10.1002/ minf.202000050 [cito:citesForInformation]

11. Chávez-Hernández AL, Sánchez-Cruz N, Medina-Franco JL (2020) Fragment library of natural products and compound databases for drug discovery. Biomolecules 10:1518. https://doi.org/10.3390/biom10111518 [cito:citesForlnformation]

12. Bento AP, Hersey A, Félix E, Landrum G, Gaulton A, Atkinson F (2020) An open source chemical structure curation pipeline using RDKit. J Cheminform 12:51. https://doi.org/10.1186/s13321-020-00456-1 [cito:usesMethodln]

13. Willighagen EL, Mayfield JW, Alvarsson J, Berg A, Carlsson L, Jeliazkova N (2017) The Chemistry Development Kit (CDK) v2.0: atom typing, depiction, molecular formulas, and substructure searching. J Cheminform 9:33. https://doi.org/10.1186/s13321-017-0220-4 [cito:usesMethodIn]

14. Djoumbou Feunang Y, Eisner R, Knox C, Chepelev L, Hastings J, Owen G (2016) ClassyFire: automated chemical classification with a comprehensive, computable taxonomy. J Cheminform 8:61. https://doi.org/10.1186/ s13321-016-0174-y [cito:usesMethodIn]

15. Bemis GW, Murcko MA (1996) The properties of known drugs. 1. Molecular frameworks. J Med Chem 39:2887-2893. https://doi.org/10.1021/ jm9602928 [cito:usesMethodln]

16. Fritsch S, Neumann S, Schaub J, Steinbeck C, Zielesny A (2019) ErtlFunctionalGroupsFinder: automated rule-based functional group detection with the Chemistry Development Kit (CDK). J Cheminform 11:37. https:// doi.org/10.1186/s13321-019-0361-8 [cito:usesMethodIn]

17. O'Boyle N, Dalke A (2018) DeepSMILES: an adaptation of SMILES for use in machine-learning of chemical structures. https://doi.org/10.26434/chemr xiv.7097960.v1 [cito:usesMethodln]

18. Hastings J, de Matos P, Dekker A, Ennis M, Harsha B, Kale N (2013) The ChEBI reference database and ontology for biologically relevant chemistry: enhancements for 2013. Nucleic Acids Res 41:D456-D463. https://doi. org/10.1093/nar/gks1146

19. Gaulton A, Hersey A, Nowotka M, Bento AP, Chambers J, Mendez D (2017) The ChEMBL database in 2017. Nucleic Acids Res 45:D945-D954. https://doi.org/10.1093/nar/gkw1074 [cito:usesDataFrom]

20. ChemAxon (2012) JChem Base was used for structure searching and chemical database access and management. http://www.chemaxon. com.

21. Schaub J, Zielesny A, Steinbeck C, Sorokina M (2020) Too sweet: cheminformatics for deglycosylation in natural products. J Cheminform 12:67. https://doi.org/10.1186/s13321-020-00467-y [cito:usesMethodln]

22. Ertl P, Roggo S, Schuffenhauer A (2008) Natural product-likeness score and its application for prioritization of compound libraries. J Chem Inf Model 48:68-74. https://doi.org/10.1021/ci700286x [cito:usesMethodIn]

23. Sorokina M, Steinbeck C (2019) NaPleS: a natural products likeness scorer — web application and database. J Cheminformatics. https://doi. org/10.1186/s13321-019-0378-z [cito:usesMethodln]

24. Kim H, Wang M, Leber C, Nothias L-F, Reher R, Kang KB, et al. (2020) NPClassifier: a Deep Neural Network-Based Structural Classification Tool for Natural Products. https://doi.org/10.26434/chemrxiv.12885494.v1 [cito:usesMethodln]

25. Kim S, Thiessen PA, Bolton EE, Chen J, Fu G, Gindulyte A (2016) PubChem Substance and Compound databases. Nucleic Acids Res 44:D1202D1213. https://doi.org/10.1093/nar/gkv951 [cito:usesMethodln]

26. React - A JavaScript library for building user interfaces. https://react js.org/. Accessed 21 Aug 2020 [cito:usesMethodln]

27. OpenChemLib (https://github.com/cheminfo/openchemlib-js). JavaScript (2020) https://github.com/cheminfo/openchemlib-js. Accessed 21 Aug 2020 [cito:usesMethodln]

28. \$bitsAllSet — MongoDB Manual. https://github.com/mongodb/docs/ blob/master/source/reference/operator/query/bitsAllset.txt. https://docs. mongodb.com/manual/reference/operator/query/bitsAllset. Accessed 21 Aug 2020 [cito:usesMethodln]

29. Ullmann (cdk 2.3 API). http://cdk.github.io/cdk/latest/docs/api/index .html. Accessed 21 Aug 2020 [cito:usesMethodln]

30. Cordella LP, Foggia P, Sansone C, Vento M (2004) A (sub)graph isomorphism algorithm for matching large graphs IEEE Trans Pattern Anal Mach Intell 26:1367-1372. [cito:usesMethodln]

31. DfPattern (cdk 2.3 API). http://cdk.github.io/cdk/latest/docs/api/index .html. Accessed 28 Sep 2020 [cito:usesMethodln]
32. Michał. LSH-based similarity search in MongoDB is faster than postgres cartridge. THE CHEMBL-OG The Organization of Drug Discovery Data. http://chembl.blogspot.com/2015/08/lsh-based-similarity-search-inmongodb.html. Accessed 21 Aug 2020 [cito:usesMethodln]

33. Ntie-Kang F, Nwodo JN, Ibezim A, Simoben CV, Karaman B, Ngwa VF (2014) Molecular modeling of potential anticancer agents from African medicinal plants. J Chem Inf Model 54:2433-2450. https://doi. org/10.1021/ci5003697

34. Ntie-Kang F, Zofou D, Babiaka SB, Meudom R, Scharfe M, Lifongo LL (2013) AfroDb: a select highly potent and diverse natural product library from African medicinal plants. PLoS ONE 8:e78085

35. Onguéné PA, Ntie-Kang F, Mbah JA, Lifongo LL, Ndom JC, Sippl W (2014) The potential of anti-malarial compounds derived from African medicinal plants, part III: an in silico evaluation of drug metabolism and pharmacokinetics profiling. Org Med Chem Lett 4:6. https://doi.org/10.1186/ s13588-014-0006-x

36. AnalytiCon Discovery, Screening Libraries. In: AnalytiCon Discovery. https ://ac-discovery.com/screening-libraries/. Accessed 16 Oct 2020

37. Pilón-Jiménez BA, Saldívar-González Fl, Díaz-Eufracio BI, Medina-Franco JL (2019) BIOFACQUIM: a Mexican compound database of natural products. Biomolecules 9:31. https://doi.org/10.3390/biom9010031

38. Dagan-Wiener A, Di Pizio A, Nissim I, Bahia MS, Dubovski N, Margulis E (2019) BitterDB: taste ligands and receptors database in 2019. Nucleic Acids Res 47:D1179-D1185. https://doi.org/10.1093/nar/gky974

39. Yabuzaki J (2017) Carotenoids Database: structures, chemical fingerprints and distribution among organisms. Database J Biol Databases Curation. https://doi.org/10.1093/database/bax004

40. Pence HE, Williams A (2010) ChemSpider: an online chemical information resource. J Chem Educ 87:1123-1124. https://doi.org/10.1021/ed100 $697 \mathrm{w}$

41. Ntie-Kang F, Amoa Onguéné P, Scharfe M, Owono LCO, Megnassan E, Meva'a Mbaze L (2014) ConMedNP: a natural product library from Central African medicinal plants for drug discovery. RSC Adv 4:409-419. https ://doi.org/10.1039/c3ra43754j

42. Bultum LE, Woyessa AM, Lee D (2019) ETM-DB: integrated Ethiopian traditional herbal medicine and phytochemicals database. BMC Complement Altern Med 19:212. https://doi.org/10.1186/s12906-019-2634-1

43. Neveu V, Moussy A, Rouaix H, Wedekind R, Pon A, Knox C (2017) Exposome-Explorer: a manually-curated database on biomarkers of exposure to dietary and environmental factors. Nucleic Acids Res 45:D979-D984. https://doi.org/10.1093/nar/gkw980

44. Wang M, Carver JJ, Phelan VV, Sanchez LM, Garg N, Peng Y (2016) Sharing and community curation of mass spectrometry data with Global Natural Products Social Molecular Networking. Nat Biotechnol 34:828. https://doi.org/10.1038/nbt.3597

45. Kang H, Tang K, Liu Q, Sun Y, Huang Q, Zhu R (2013) HIM-herbal ingredients in vivo metabolism database. J Cheminform 5:28. https://doi. org/10.1186/1758-2946-5-28

46. Ye H, Ye L, Kang H, Zhang D, Tao L, Tang K (2011) HIT: linking herbal active ingredients to targets. Nucleic Acids Res 39:D1055-D1059 https:// doi.org/10.1093/nar/gkq1165

47. NDOFINE Chemical Company. http://www.indofinechemical.com/Media/ sdf/sdf files.aspx. Accessed 16 Oct 2019

48. Zhang R, Lin J, Zou Y, Zhang X-J, Xiao W-L (2019) Chemical space and biological target network of anti-inflammatory natural products, J Chem Inf Model 59:66-73. https://doi.org/10.1021/acs.jcim.8b00560

49. Vetrivel U, Subramanian N, Pilla K (2009) InPACdb—Indian plant anticancer compounds database. Bioinformation 4:71-74

50. InterBioScreen | Natural Compounds. https://www.ibscreen.com/natur al-compounds. Accessed 9 Oct 2019

51. Lichen Database. In: MTBLS999: A database of high-resolution MS/MS spectra for lichen metabolites. https://www.ebi.ac.uk/metabolights/ MTBLS999. Accessed 16 Oct 2019

52. Gentile D, Patamia V, Scala A, Sciortino MT, Piperno A, Rescifina A (2020) Putative inhibitors of SARS-CoV-2 main protease from a library of marine natural products: a virtual screening and molecular modeling study. Marine Drugs 18:225. https://doi.org/10.3390/md18040225

53. Derese S, Oyim J, Rogo M, Ndakala A (2015) Mitishamba database: a web based in silico database of natural products from Kenya plants. Nairobi, University of Nairobi 
54. Ntie-Kang F, Telukunta KK, Döring K, Simoben CV, Moumbock AF, Malange YI (2017) NANPDB: a resource for natural products from Northern African sources. J Nat Prod 80:2067-2076. https://doi.org/10.1021/acs. jnatprod.7b00283

55. Compound Sets-NCI DTP Data-National Cancer Institute-Confluence Wiki. https://wiki.nci.nih.gov/display/NCIDTPdata/Compound+Sets. Accessed 18 Oct 2019

56. Mangal M, Sagar P, Singh H, Raghava GPS, Agarwal SM (2013) NPACT: naturally occurring plant-based anti-cancer compound-activity-target database. Nucleic Acids Res 41:D1124-D1129. https://doi.org/10.1093/ nar/gks 1047

57. Zeng X, Zhang P, He W, Qin C, Chen S, Tao L (2018) NPASS: natural product activity and species source database for natural product research, discovery and tool development. Nucleic Acids Res 46:D1217-D1222. https://doi.org/10.1093/nar/gkx1026

58. Choi H, Cho SY, Pak HJ, Kim Y, Choi J, Lee YJ (2017) NPCARE: database of natural products and fractional extracts for cancer regulation. J Cheminformatics 9:2. https://doi.org/10.1186/s13321-016-0188-5

59. Tomiki T, Saito T, Ueki M, Konno H, Asaoka T, Suzuki R (2006) RIKEN natural products encyclopedia (RIKEN NPEdia), a chemical database of RIKEN natural products depository (RIKEN NPDepo). J Comput Aid Chem 7:157-162

60. Ntie-Kang F, Onguéné PA, Fotso GW, Andrae-Marobela K, Bezabih M, Ndom JC (2014) Virtualizing the p-ANAPL library: a step towards drug discovery from African medicinal plants. PLoS ONE 9:e90655. https://doi. org/10.1371/journal.pone.0090655

61. Rothwell JA, Perez-Jimenez J, Neveu V, Medina-Remón A, M'Hiri N, García-Lobato P (2013) Phenol-Explorer 3.0: a major update of the Phenol-Explorer database to incorporate data on the effects of food processing on polyphenol content. Database. https://doi.org/10.1093/ database/bat070

62. Sawada Y, Nakabayashi R, Yamada Y, Suzuki M, Sato M, Sakata A (2012) RIKEN tandem mass spectral database (ReSpect) for phytochemicals: a plant-specific MS/MS-based data resource and database. Phytochemistry 82:38-45. https://doi.org/10.1016/j.phytochem.2012.07.007

63. Hatherley R, Brown DK, Musyoka TM, Penkler DL, Faya N, Lobb KA (2015) SANCDB: a South African natural compound database. J Cheminformatics 7:29. https://doi.org/10.1186/s13321-015-0080-8

64. Davis GDJ, Vasanthi AHR (2011) Seaweed metabolite database (SWMD): a database of natural compounds from marine algae. Bioinformation 5:361-364.
65. Specs. Compound management services and research compounds for the life science industry. https://www.specs.net/index.php. Accessed 16 Oct 2019

66. Fischedick JT, Johnson SR, Ketchum REB, Croteau RB, Lange BM (2015) NMR spectroscopic search module for Spektraris, an online resource for plant natural product identification-Taxane diterpenoids from Taxus $\times$ media cell suspension cultures as a case study. Phytochemistry 113:87-95. https://doi.org/10.1016/j.phytochem.2014.11.020

67. Moumbock AFA, Gao M, Qaseem A, Li J, Kirchner PA, Ndingkokhar B (2020) StreptomeDB 3.0: an updated compendium of streptomycetes natural products. Nucleic Acids Res. https://doi.org/10.1093/nar/gkaa868

68. TCMID: traditional Chinese medicine integrative database for herb molecular mechanism analysis. https://www.ncbi.nlm.nih.gov/pmc/artic les/PMC3531123/. Accessed 29 Apr 2019

69. Tung C-W, Lin Y-C, Chang H-S, Wang C-C, Chen I-S, Jheng J-L (2014) TIPdb-3D: the three-dimensional structure database of phytochemicals from Taiwan indigenous plants. Database. https://doi.org/10.1093/datab ase/bau055

70. ünthardt BF, Hollender J, Hungerbühler K, Scheringer M, Bucheli TD (2018) Comprehensive toxic plants-phytotoxins database and its application in assessing aquatic micropollution potential. J Agric Food Chem 66:7577-7588. https://doi.org/10.1021/acs.jafc.8b01639

71. UEFS Natural Products. http://zinc12.docking.org/catalogs/uefsnp. Accessed 6 Nov 2019

72. Gu J, Gui Y, Chen L, Yuan G, Lu H-Z, Xu X (2013) Use of natural products as chemical library for drug discovery and network pharmacology. PLoS ONE 8:e62839. https://doi.org/10.1371/journal.pone.0062839

73. Nguyen-Vo T-H, Le T, Pham D, Nguyen T, Le P, Nguyen A (2019) VIETHERB: a database for Vietnamese herbal species. J Chem Inf Model 59:1-9. https ://doi.org/10.1021/acs.jcim.8b00399

74. Sterling T, Irwin JJ (2015) ZINC 15-ligand discovery for everyone. J Chem Inf Model 55:2324-2337. https://doi.org/10.1021/acs.jcim.5b00559

\section{Publisher's Note}

Springer Nature remains neutral with regard to jurisdictional claims in published maps and institutional affiliations.
Ready to submit your research? Choose BMC and benefit from:

- fast, convenient online submission

- thorough peer review by experienced researchers in your field

- rapid publication on acceptance

- support for research data, including large and complex data types

- gold Open Access which fosters wider collaboration and increased citations

- maximum visibility for your research: over $100 \mathrm{M}$ website views per year

At BMC, research is always in progress.

Learn more biomedcentral.com/submissions 